\title{
Effect of Gold Nanoparticle Radiosensitization on Plasmid DNA Damage Induced by High-Dose-Rate Brachytherapy
}

This article was published in the following Dove Press journal: International Journal of Nanomedicine

\section{Katsunori Yogo $\mathbb{D}^{1}$ \\ Masaki Misawa ${ }^{2}$ \\ Morihito Shimizu \\ Hidetoshi Shimizu ${ }^{3}$ \\ Tomoki Kitagawa ${ }^{3}$ \\ Ryoichi Hirayama (D) ${ }^{4}$ \\ Hiromichi Ishiyama ${ }^{5}$ \\ Takako Furukawa' \\ Hiroshi Yasuda ${ }^{6}$}

'Nagoya University Graduate School of Medicine, Nagoya, Aichi, Japan; ${ }^{2}$ Health and Medical Research Institute, National Institute of Advanced Industrial Science \& Technology (AIST), Tsukuba, Ibaraki, Japan; ${ }^{3}$ Department of Radiation Oncology, Aichi Cancer Center Hospital, Nagoya, Aichi, Japan; ${ }^{4}$ National Institute of Radiological Sciences (NIRS), National Institutes for Quantum and Radiological Science and Technology (QST), Chibashi, Chiba, Japan; ${ }^{5} \mathrm{Graduate}$ School of Medical Science, Kitasato University, Sagamihara, Kanagawa, Japan; ${ }^{6}$ Department of Radiation Biophysics, Research Institute for Radiation Biology and Medicine, Hiroshima University, Hiroshima, Japan
Correspondence: Katsunori Yogo Nagoya University Graduate School of Medicine, I-I-20 Daiko-Minami, HigashiKu, Nagoya, Aichi 46I-8673, Japan

Tel +8I 527191103

Fax +8I 527193172

Email yogo@met.nagoya-u.ac.jp
Purpose: Gold nanoparticles (AuNPs) are candidate radiosensitizers for medium-energy photon treatment, such as $\gamma$-ray radiation in high-dose-rate (HDR) brachytherapy. However, high AuNP concentrations are required for sufficient dose enhancement for clinical applications. Here, we investigated the effect of positively $(+)$ charged AuNP radiosensitization of plasmid DNA damage induced by 192Ir $\gamma$-rays, and compared it with that of negatively $(-)$ charged AuNPs.

Methods: We observed DNA breaks and reactive oxygen species (ROS) generation in the presence of AuNPs at low concentrations. pBR322 plasmid DNA exposed to $64 \mathrm{ng} / \mathrm{mL}$ AuNPs was irradiated with 192Ir $\gamma$-rays via HDR brachytherapy. DNA breaks were detected by observing the changes in the form of the plasmid and quantified by agarose gel electrophoresis. The ROS generated by the AuNPs were measured with the fluorescent probe sensitive to ROS. The effects of positively $(+)$ and negatively $(-)$ charged AuNPs were compared to study the effect of surface charge on dose enhancement.

Results: + AuNPs at lower concentrations promoted a comparable level of radiosensitization by producing both single-stranded breaks (SSBs) and double-stranded breaks (DSBs) than those used in cell assays and Monte Carlo simulation experiments. The dose enhancement factor (DEF) for +AuNPs was $1.3 \pm 0.2$ for SSBs and $1.5 \pm 0.4$ for DSBs. The ability of + AuNPs to augment plasmid DNA damage is due to enhanced ROS generation. While -AuNPs generated similar ROS levels, they did not cause significant DNA damage. Thus, dose enhancement using low concentrations of +AuNPs presumably occurred via DNA binding or increasing local +AuNP concentration around the DNA.

Conclusion: +AuNPs at low concentrations displayed stronger radiosensitization compared to -AuNPs. Combining +AuNPs with 192Ir $\gamma$-rays in HDR brachytherapy is a candidate method for improving clinical outcomes. Future development of cancer cell-specific +AuNPs would allow their wider application for HDR brachytherapy.

Keywords: gold nanoparticles, high-dose-rate brachytherapy, DNA damage, radiosensitization, ${ }^{192} \operatorname{Ir} \gamma$-rays, positively charged nanoparticles

\section{Introduction}

Recent advances in radiation therapy techniques have enabled the delivery of increased radiation doses to tumors. For example, a highly active ${ }^{192} \operatorname{Ir} \gamma$-ray source in high-dose-rate (HDR) brachytherapy delivers a higher and a localized dose to tumors within a shorter treatment time and with fewer fractions, than that by external beam therapy. ${ }^{1}$ Using a wire inside an implanted flexible catheter tube or solid tube, the Ir source is placed for treatment using an HDR treatment machine. 
The treatment machine can deliver the source remotely using computer-controlled methods (remote afterloading brachytherapy system).

Cervix cancer brachytherapy is an HDR brachytherapy that is used extensively in clinical settings and exhibits positive clinical outcomes. Several irradiation techniques and dose optimization methods have been developed over the past decade. ${ }^{2-6}$ Despite this, normal tissue injury, such as rectal tissue damage following radiation treatment of cervical cancer, still prevents the delivery of optimal doses, which is detrimental to treatment outcomes. Marginal recurrence often occurs around the radiated fields near the tumors where the availability of radiation tends to reduce such as in case of advanced cervical cancers. Thus, radiation sensitization agents that modify and enhance the local dose are necessary to deliver a sufficient dose to tumors, including its marginal regions.

Gold nanoparticles (AuNPs) are candidate radiosensitization agents for radiation therapy. ${ }^{7,8}$ AuNPs are particles with sizes on the nanometer scale. As with other high- $Z$ materials, Gold $(\mathrm{Au})$ is relatively safe for use in vivo. AuNPs cause dose enhancement by increasing photoelectric absorption, resulting in increased reactive oxygen species (ROS) generation. ${ }^{9}$ We propose a combination of HDR brachytherapy and AuNPs as a radiosensitizer, which can be an effective treatment against potential metastasis around primary lesions.

Previous studies on AuNP radiosensitization combined with radiation therapy mainly focused on the use of external beams, such as $\mathrm{kV}$ or MV X-rays. ${ }^{10-14}$ In the past few years, significant progress has been reported on the use of AuNPs combined with brachytherapy. ${ }^{15}$ These studies have proposed the substitution of the radioactive seeds (millimeter-sized) used in low-dose-rate brachytherapy with the injection of radioactive or non-radioactive AuNPs to enhance the effect of radiation. This treatment method has been named "nanobrachytherapy," and is used for treating prostate cancer with low-energy photons such as ${ }^{125} \mathrm{I}$ and ${ }^{103} \mathrm{Pd}$.

The dose enhancement effects by AuNPs are predicted to be larger for low-energy photons (optimal energy $~ 40$ $\mathrm{keV}$ ). However, the use of low-energy photons in radiation therapy is limited to the treatment of superficial tumors. Low-dose-rate (LDR) brachytherapy uses low-energy photons and can provide higher values of DEF compared with HDR sources. ${ }^{15}$ The use of an LDR source is rather limited in the current radiation therapy, such as in that for prostate cancers. In contrast, high-energy photons (MV
X-rays from linear accelerators) are dominantly and widely used in the current radiation therapy. Despite this, the dose enhancement that occurs by using AuNPs with high-energy photons is predicted to be much smaller than that observed with lower energy photons. This study aimed to explore AuNP sensitization combined with HDR brachytherapy, which uses medium-energy photons. These photons are therefore predicted to achieve a better balance between clinical application and AuNP enhancement. Several studies on dose enhancement by AuNPs with HDR $\left({ }^{192} \mathrm{Ir}\right)$ brachytherapy have been previously reported. Shahhoseini et al reported that the dose enhance-

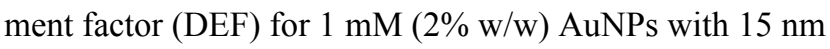
was approximately 1.6 for cancer cell lines, as studied via cell survival assays. ${ }^{16}$ Physical dose enhancements by AuNPs in HDR brachytherapy were studied by Monte Carlo (MC) calculations in previous studies. ${ }^{17-19}$ Cho et al demonstrated a DEF of $\sim 1.3$ in tumors administered with $30 \mathrm{mg} / \mathrm{g}$ AuNPs, as determined by MC calculations. ${ }^{17}$ Lechtman et al revealed that the administration of $\sim 100 \mathrm{mg} / \mathrm{g}$ AuNPs is necessary for tumors treated with ${ }^{192}$ Ir $\gamma$-rays to achieve a dose enhancement of double the prescribed dose. ${ }^{18}$ Zabihzadeh et al reported that the DEF was predicted to be approximately 1.2 upon administration of uniformly distributed AuNPs (30 mg/g). ${ }^{19}$ These studies using MC calculations revealed that a high concentration of AuNPs $(10-100 \mathrm{mg} / \mathrm{g})$ is necessary in tumors treated with ${ }^{192}$ Ir $\gamma$-rays, which is an unrealistic concentration for clinical cases. The $50 \%$ lethal dose (LD50) of $\sim 3.2 \mathrm{~g} / \mathrm{kg}$ has been reported for mice. ${ }^{7}$ Thus, the concentration of AuNPs necessary to produce significant dose enhancement must be reduced for clinical applications.

Radiation is known to injure cancer cells by triggering DNA damage. Therefore, revealing whether the AuNP affects radiation-induced events at the DNA level is an important step for clinical applications. For this purpose, plasmid DNA assays were widely performed..$^{20-24}$ Merits of use of the plasmid include high sensitivity and easy detection of DNA damage. There are several studies on plasmid damage enhancement by AuNP with low-energy $(\mathrm{kV})$ photons. ${ }^{20-24}$ However, the ability of AuNPs to sensitize cells to ${ }^{192} \operatorname{Ir} \gamma$-ray-induced DNA damage has not yet been studied. Dose enhancement by AuNPs is known to be dependent on photon energy and the energy spectrum. Previous studies have reported that the energy of photons has a major influence on the radiosensitizing power of AuNPs. ${ }^{7,8}$ The AuNPs sensitization on ${ }^{192}$ Ir $\gamma$-rayinduced DNA damage must be confirmed experimentally 
before clinical application, as the experimental data are limited compared to the studies on external beam therapy. Here, we investigated the effect of AuNP radiosensitization on plasmid DNA damage induced by $\gamma$-rays via HDR brachytherapy. We used ${ }^{192}$ Ir $\gamma$-rays with medium-energy photons $(380 \mathrm{keV})$, which are clinically used in HDR brachytherapy to study DNA damage enhancement in terms of both single-stranded and double-stranded breaks. To reduce the concentration of AuNPs required, we used positively charged AuNPs to target negatively charged DNA. Furthermore, we studied the generation of ROS in the presence of AuNPs with fluorescent probes sensitive to ROS. The effects of positively $(+)$ and negatively (-) charged AuNPs were compared to study the effects of the surface charge on dose enhancement.

\section{Materials and Methods}

\section{Gold Nanoparticles and Characterization}

Positively charged gold nanoparticles (+AuNPs) were prepared and synthesized through the chemical reduction of tetrachloroaurate $\left(\mathrm{HAuCl}_{4}\right)$ in the presence of the 2-aminoethanthiol (AET) as described earlier. ${ }^{25}$ Briefly, a mixture of $\mathrm{HAuCl}_{4}$ and AET at a molar ratio of $1: 1.5$ was added to $0.1 \mathrm{mM}$ sodium borohydrate $\left(\mathrm{NaBH}_{4}\right)$ and reactions were terminated by adding $10 \mathrm{mM}$ hydrogen peroxide. Following synthesis, the AuNPs were dialyzed to remove unreacted chemicals.

Positive charges were applied by modifying the AuNP surfaces with amine groups. +AuNPs were used at a final concentration of $64 \mathrm{ng} / \mathrm{mL}$. We also used +AuNPs with a diameter of $1.4 \mathrm{~nm}$ (Cat\# 2022, Nanoprobes, Yaphank, NY, USA). Negatively charged AuNPs (-AuNPs) with a diameter of $30 \mathrm{~nm}$ were also used (Cat\#EM.GC30, British BioCell International Co. Ltd., Cardiff, United Kingdom). Negative charges were applied by citrate ligands, which remained after synthesis. It was comparable in terms of size and shape to AET. These negative charges realize stable dispersion of -AuNPs.

AuNPs were characterized using absorption spectroscopy (SynergyHTX, BioTek, VT, USA) and the dynamic light scattering method (ZetaSizer Nano-S, Malvern Instruments, Malvern, UK). In the spectrum, AuNPs produce a characteristic absorption peak around $530 \mathrm{~nm}$. The height of the peak represents the size concentration of AuNPs. ${ }^{21}$ AuNPs were also observed by field emission scanning electron microscopy (FESEM) (S-4800, Hitachi High-Tech, Ibaraki, Japan).
Zeta potentials reflecting the surface charge of the AuNPs were measured using ZetaSizer Nano-S. The concentrations of the AuNPs were analyzed by adding AuNP solutions to a glass tube and measuring the dry weight of colloids after evaporating the solutions. The concentration was independently measured by absorbance at $530 \mathrm{~nm}$ and was confirmed as being consistent with the weight measurements.

\section{Plasmid DNA and Reagents}

The 4.3-kbp pBR322 plasmid DNA (Cat\# 319-00444, Nippon Gene Co., Ltd., Tokyo, Japan) suspended in Trisethylenediaminetetraacetic acid (TE) buffer was precipitated with ethanol to remove the buffer. ${ }^{26,27}$ The DNA in ultra-pure water was aliquoted and stored at $-20^{\circ} \mathrm{C}$ until use. The DNA concentration was quantified by absorption at $260 \mathrm{~nm}$ using a spectrometer (NanoDrop, Thermo Fisher Scientific, Waltham, MA, US).

For irradiation using $\gamma$-rays in HDR brachytherapy, 500 ng DNA was mixed in $20 \mu \mathrm{L}$ buffer with $64 \mathrm{ng} / \mathrm{mL}$ AuNPs and placed in a $0.5 \mathrm{~mL}$ polypropylene microtube. A low concentration of TE buffer $(0.25 \mathrm{mM}$ Tris, $0.025 \mathrm{mM}$ ethylenediaminetetraacetic acid (EDTA), $\mathrm{pH}$ 7.5) was used for the subsequent DNA assays, as appropriate. ${ }^{21}$ Damage yields in the plasmid assay are highly dependent on the scavenging capacity of the prepared buffer. Tris buffer $(0.25 \mathrm{mM})$ provided only a weak scavenging capacity. This buffer was selected to provide sensitivity to damage, allowing for radiation exposure via therapeutic HDR brachytherapy to be completed within a practical time period.

\section{Irradiation Conditions}

The sample tubes were fixed to a holder custom-made with water-equivalent plastics (Tough Water Phantom, Kyoto Kagaku Co., Ltd., Kyoto, Japan). The sample tubes were irradiated from the bottom under aerobic conditions with an ${ }^{192}$ Ir $\gamma$-ray at Aichi Cancer Center (Figure 1). An ${ }^{192} \mathrm{Ir}$ source (Nucletron mHDR-v2, $\gamma$ rays; mean energy: $0.355 \mathrm{MeV}$; half-life: 73.81 days; dimensions: $3.5 \mathrm{~mm} \times 0.6 \mathrm{~mm}$ ) was driven into the catheters placed beneath the holder (Figure 1A). A single Ir source was connected to the wire driven using an HDR brachytherapy unit (microSelectron HDRV3, Nucletron). The source moves in two tubes (brachytherapy applicator indicated in blue in Figure 1A), and stopped at different positions (indicated using yellow arrows), to provide a uniform dose. The delivered dose 

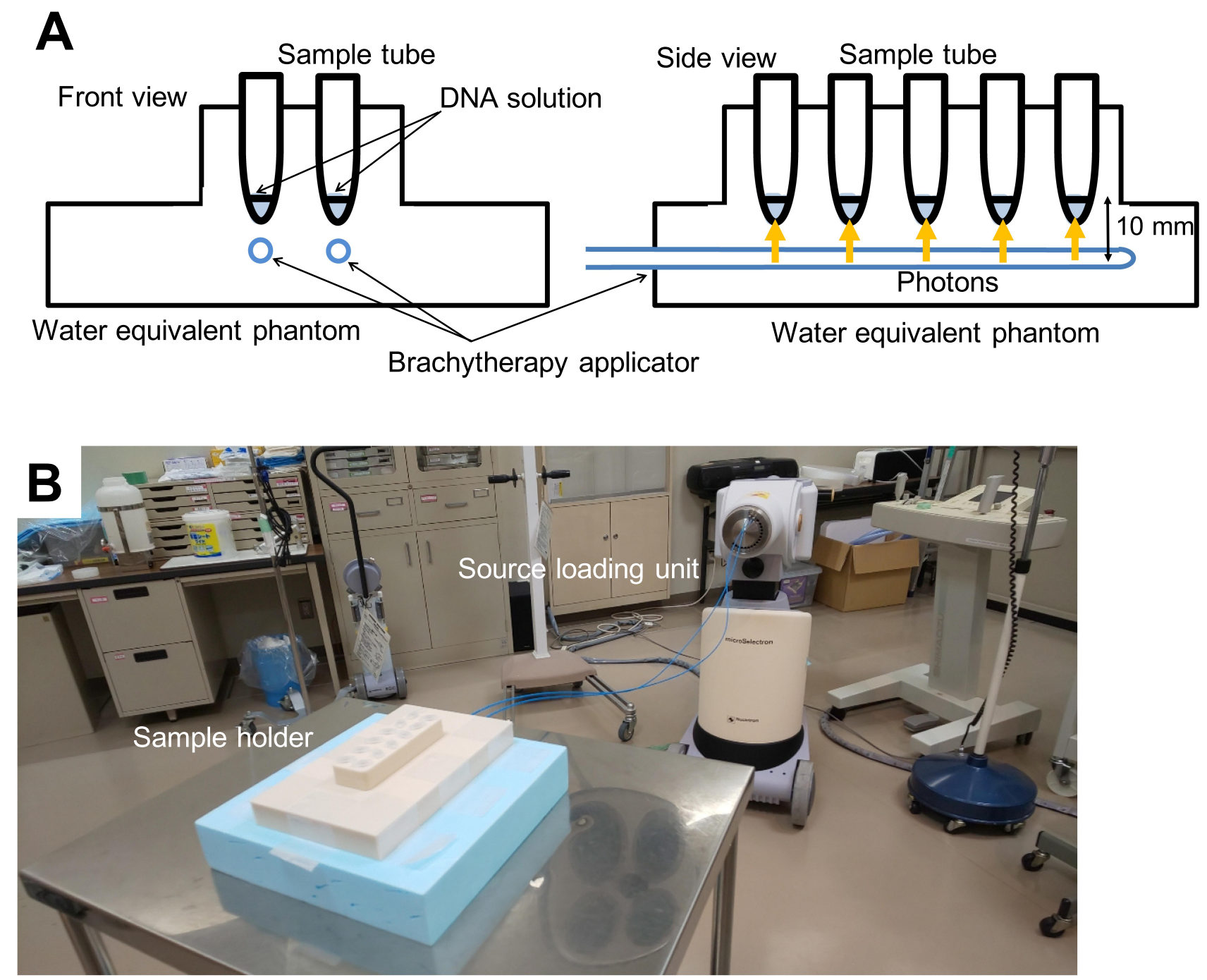

Figure I Experimental setup. (A) Schematic and (B) photo of the plastic phantom for the sample holder and ${ }^{192}$ Ir source loading unit.

was controlled by the source positions and dwell times using the loading unit. Doses of 4-20 Gy were delivered to the samples.

Dose calculations were performed with treatment planning systems (Oncentra v4.3, Nucletron) considering the thickness of the sample tube. Doses were delivered to cover the top of the DNA solution in the tubes with 4 Gy isodose lines located at $\sim 10 \mathrm{~mm}$ from the ${ }^{192} \mathrm{Ir}$ source and defined as the reference point. ${ }^{28,29}$ An air kerma

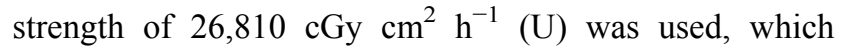
corresponds to $242 \mathrm{GBq}$. A dose rate was approximately $5 \mathrm{~Gy} / \mathrm{min}$ at the reference point.

The sample irradiations were performed within 2-3 hours on the same day. The samples were stored at $4^{\circ} \mathrm{C}$ until electrophoresis. The electrophoresis was performed within a few hours of irradiation.

\section{Plasmid DNA Form Analysis}

Breaks in the DNA were detected as form changes in the plasmids and quantified by subsequent electrophoresis on agarose gels. The native plasmid forms a supercoiled (SC) structure, similar to a small ring twisting. When DNA is irradiated, the phosphate backbone is cut, which results in the formation of single-stranded breaks (SSBs), SC plasmid was restructured into an open circular (OC) form, similar to a small ring relaxing and opening. If the phosphate backbone in both strands is excised, which results in the formation of double-stranded breaks (DSBs), the plasmid DNA changes into a linear (L) structure, like a single string. For this assay, two SSBs in proximity within approximately six base pairs were detected as an L structure. ${ }^{30}$ Samples were electrophoresed in $1 \%(\mathrm{w} / \mathrm{v})$ agarose gel in TBE buffer $(44.5 \mathrm{mM}$ of Tris-borate with 
$\mathrm{pH} 8.4$ and $1 \mathrm{mM}$ of EDTA) at $25 \mathrm{~V}$ for $5 \mathrm{~h}$ at room temperature. $^{21-33}$ The gels were stained with ethidium bromide $(1 \mu \mathrm{g} / \mathrm{mL})$ for over $20 \mathrm{~h}$ and washed with pure water for $1 \mathrm{~h}$. The gel images were captured using a highsensitive imager (ImageQuant LAS 4010, GE Healthcare, Chicago IL, US) under UV illumination. The fluorescence intensities of the three DNA bands were measured using an image analysis software (ImageQuant TL, GE Healthcare, Chicago IL, US). The intensity of the SC DNA bands was corrected by multiplying with a factor of 1.42, considering the differences in staining of the DNA forms. $^{34}$ The ratios between the amounts of SC and L DNA forms and the total amount of DNA were quantified according to the intensity of each DNA band.

\section{Calculation of SSB and DSB Yields}

The chemical yields of SSBs and DSBs caused by ${ }^{192} \operatorname{Ir} \gamma$ ray irradiation were calculated as described previously. $^{20,27}$ The SSB yield was calculated by fitting the data plotted as the logarithm of the fraction of SC plasmids as a function of the dose. The DSB yield was determined from the relationship between the fraction of $\mathrm{L}$ plasmids and the dose by fitting the data with a linear function. The SSB and DSB yields were derived by dividing each slope by the DNA mass $\left(650 \mathrm{~g} \mathrm{~mol}^{-1} \mathrm{bp}^{-1} \times\right.$ number of base pairs in the plasmid) and expressed in units of $\mathrm{Gy}^{-1} \mathrm{Da}^{-1}$, where Dalton (Da) is equivalent to the atomic mass unit. The DEFs used to measure the radiation sensitization effect were calculated as follows: (break yield in tested AuNP solution)/(break yield in control without AuNPs). ${ }^{16,17,19,21}$ Statistical analysis was performed by conducting the Tukey-Kramer test. Values were considered statistically significant at $\mathrm{P}<0.05$.

\section{Measurements of ROS Yield}

The ROS yields by the AuNPs were measured with the fluorescent probe sensitive to ROS generation, as previously described. ${ }^{9}$ We used the fluorescent probe 2-[6-(4-amino) phenoxy-3H-xanthen-3-on-9-yl] benzoic acid (APF) (Sekisui Medical Co., Ltd., Tokyo, Japan, Excitation: $490 \mathrm{~nm}$, Emission: $515 \mathrm{~nm}$ ), which is particularly sensitive to hydroxyl radical $(\bullet \mathrm{OH})$. The samples with $10 \mu \mathrm{M}$ fluorescent probe and $64 \mathrm{ng} / \mathrm{mL}$ AuNPs were placed in the sample tube. The samples were irradiated by the ${ }^{192}$ Ir $\gamma$-rays in the holder via the same procedure described for plasmid DNA irradiation. After irradiation, the brightness of the samples was measured with a plate reader (Synergy HTX, BioTek Instruments,
Inc., VT, USA) and the results were compared. Quenching of APF by AuNPs in dispersion was not considered, because the concentration of APF was much higher than those of AuNPs in the assays.

\section{Results}

\section{AuNP Characterization}

We characterized the positively charged AuNPs synthesized in this study. The AuNP size, monodispersity, concentration, and Zeta potential were examined.

Figure 2A shows part of a TEM image of a synthesized + AuNP. The AuNP showed a generally circular shape and had a size of approximately $30 \mathrm{~nm}$. Figure $2 \mathrm{~B}$ shows the absorbance spectrum of the AuNPs for visible light. Characteristic absorbance peaks of around $530 \mathrm{~nm}$ were seen for synthesized $30 \mathrm{~nm}+$ AuNPs. The diameters were $27.7 \pm 13.9 \mathrm{~nm}$ (mean \pm SD) for synthesized + AuNPs and $32.9 \pm 17.7 \mathrm{~nm}$ for - AuNPs. The diameter of the $1.4 \mathrm{~nm}$
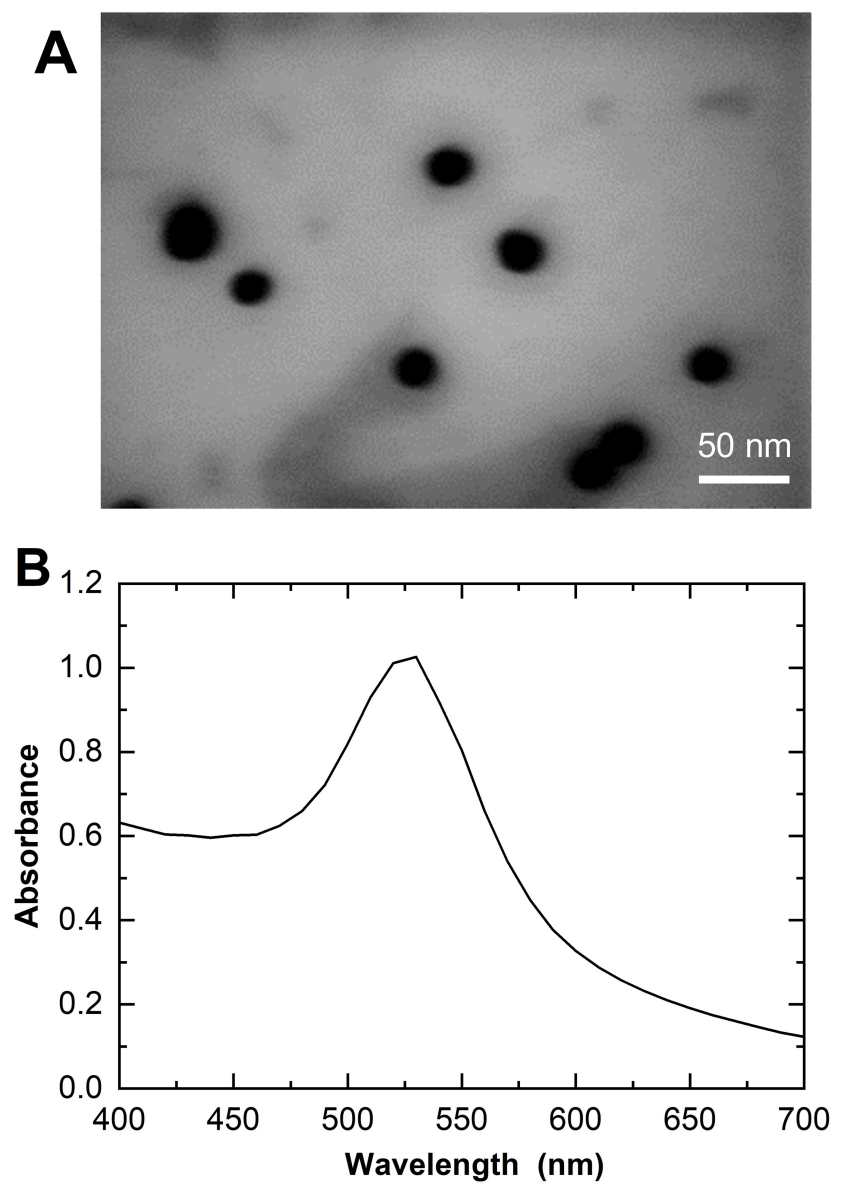

Figure 2 Characterization of positively charged gold nanoparticles (+AuNPs). (A) Transmission electron microscopy (TEM) image of 30-nm diameter +AuNPs, recorded at 400,000x magnification. Scale bar; $50 \mathrm{~nm}$. (B) Absorbance measurement of 30-nm diameter +AuNPs at visible wavelengths, including the characteristic absorbance peak at around $530 \mathrm{~nm}$. 
(nominal) +AuNPs was found to be $64.4 \pm 36.2 \mathrm{~nm}$. This was much larger than the nominal size $(1.4 \mathrm{~nm})$, presumably due to aggregations. This was further confirmed via a TEM image of the $1.4 \mathrm{~nm}+\mathrm{AuNP}$ (data not shown). The Zeta potentials were $+30 \pm 11.2 \mathrm{mV}$ for synthesized + AuNPs and $+14.9 \pm 8.5 \mathrm{mV}$ for $1.4 \mathrm{~nm}+$ AuNPs, respectively.

\section{Dose Distribution}

Figure 3 shows the dose distribution for the sample tube stand on the holder: the ${ }^{192} \operatorname{Ir}$ source was used and the dose was calculated via a treatment planning system. The top of the DNA solution in the tubes $(\sim 10 \mathrm{~mm}$ away from the source center) were covered with isodose lines of $4 \mathrm{~Gy}$. The dose was uniformly delivered to the sample tubes. The dose deviations of each sample were within $6 \%$. We irradiated DNA sample tubes as tumor targets, mimicking patient treatments. Dose delivery of HDR sources is much more localized and provides a higher dose than high-energy external beams; thus, the HDR source causes lesser side effects to normal tissues that surround the target, if the source is correctly placed in a planned position.

\section{Radiosensitization for Plasmid DNA Damage}

Figure 4 shows the fraction of SC plasmid as a function of the delivered doses. The fraction of the SC plasmid decreased with the dose in the control group, indicating that $\gamma$-ray irradiation increased the occurrence of SSBs. In the presence of +AuNPs (both $1.4 \mathrm{~nm}$ and $30 \mathrm{~nm}$ ), the decrease in the SC plasmid was much higher than that observed in the control group (without AuNPs). In contrast, the decrease in the SC plasmid in the presence of

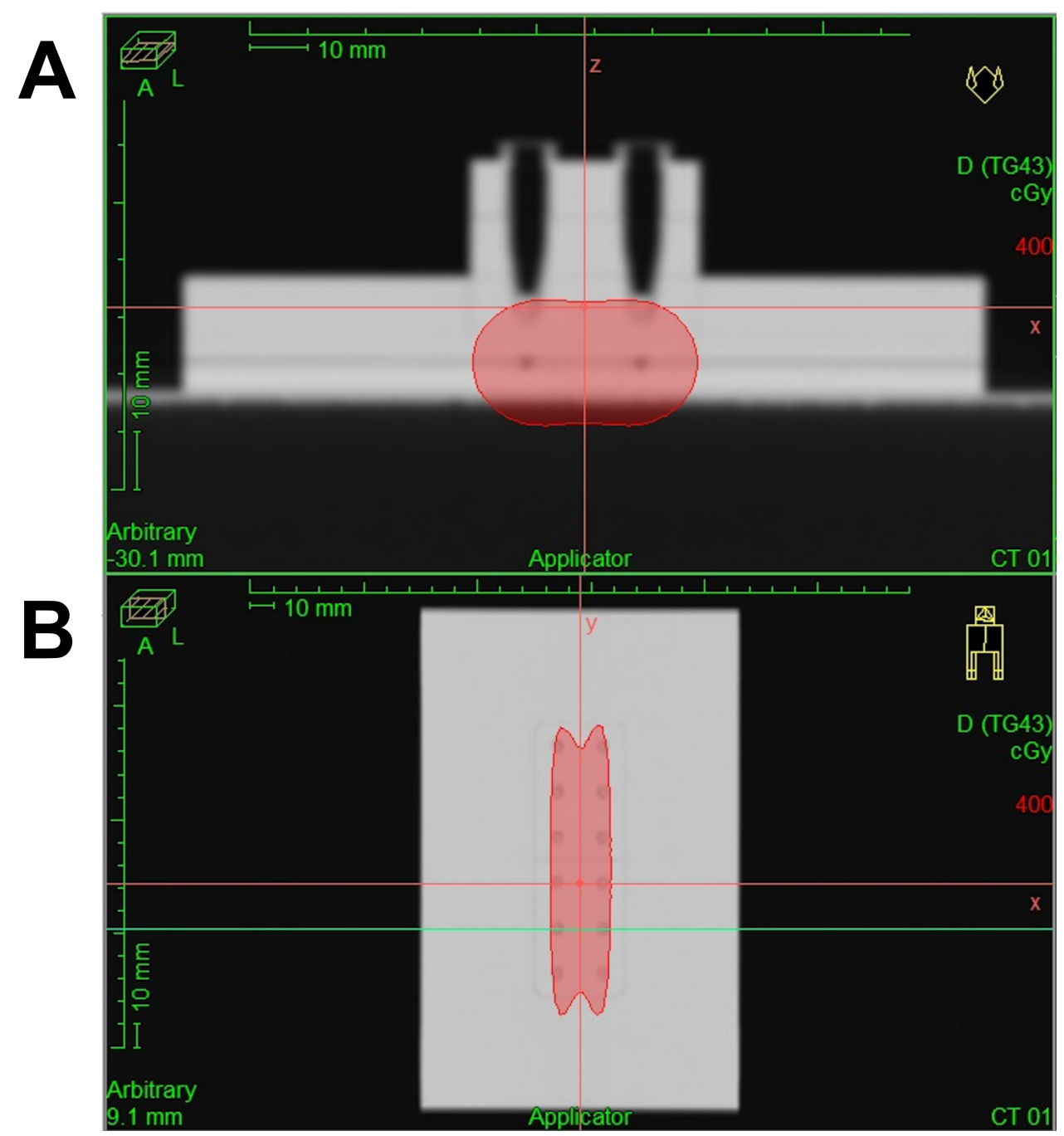

Figure 3 Dose distribution delivered around sample tube irradiated with ${ }^{192}$ Ir source. Dose distribution (4 Gy) is presented in color wash (red) from the front view (A) and the bottom view (B). Dose distribution was calculated with the treatment planning system. 


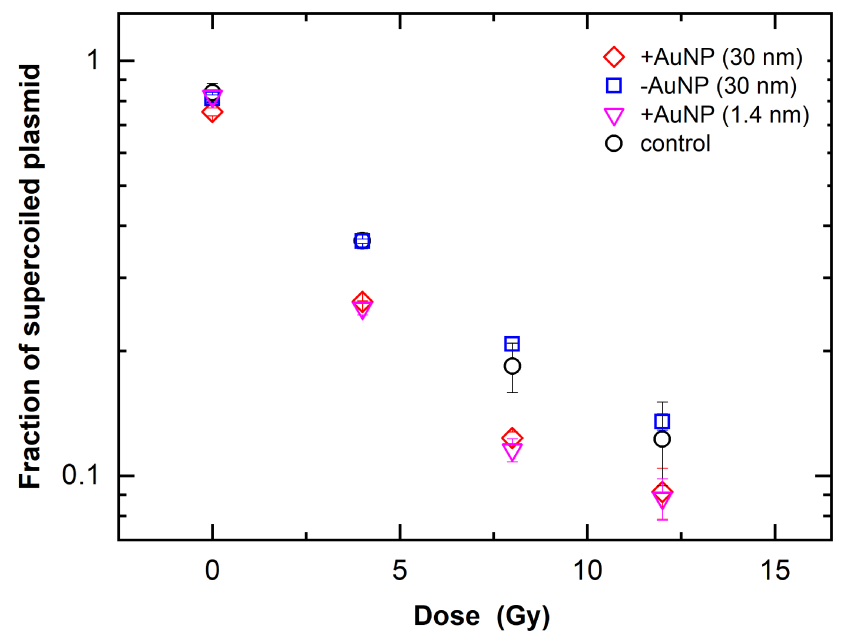

Figure 4 Supercoiled plasmid fraction as a function of radiation dose irradiated with the ${ }^{192} \mathrm{Ir}$ source in the presence of gold nanoparticles (AuNPs).

-AuNPs was not significantly different compared to that observed in the control group.

Figure 5 shows the fraction of $\mathrm{L}$ plasmids as a function of the delivered dose. The fraction of L plasmids increased with the dose in the control, indicating an increase in DSBs as a result of $\gamma$-ray irradiation. This increase in DSBs was further increased in the presence of $30-\mathrm{nm}$ diameter +AuNPs. Meanwhile, no significant changes were observed in the sample in the presence of -AuNPs and $1.4 \mathrm{~nm}+$ AuNPs. SSB and DSB yields, calculated as $\mathrm{G}$ values, were summarized for AuNPs (Table 1). These values were compared with those in the control group. The DEFs were also obtained for +AuNPs and -AuNPs relative to the control.

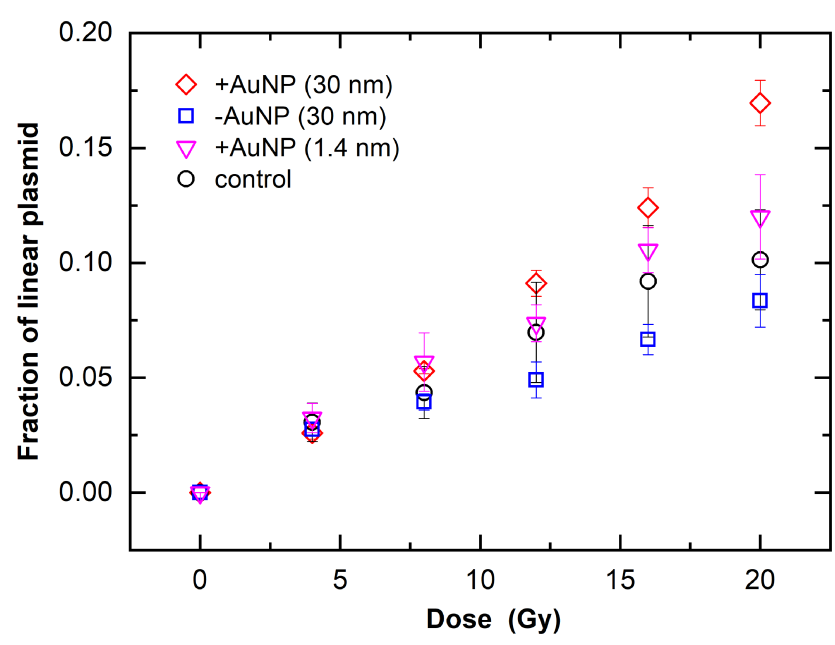

Figure $\mathbf{5}$ Linear plasmid fraction as a function of radiation dose irradiated with the ${ }^{192} \mathrm{Ir}$ source in the presence of gold nanoparticles (AuNPs).
Table I Chemical Yields of Single (SSBs) and Double-Stranded Breaks (DSBs) in ${ }^{192}$ Ir-Irradiated Samples in the Presence of Gold Nanoparticles (AuNPs) and Corresponding Dose Enhancement Factors. Data are Presented as the Mean \pm Standard Deviations from Three Independent Experiments

\begin{tabular}{|l|l|l|l|l|}
\hline \multirow{2}{*}{$\begin{array}{l}\text { Experimental } \\
\text { Conditions }\end{array}$} & \multicolumn{2}{|l|}{ Yields (Breaks per Da per Gy)* } & \multicolumn{2}{l|}{$\begin{array}{l}\text { Dose Enhancement } \\
\text { Factor** }\end{array}$} \\
\cline { 2 - 5 } & SSB & DSB & SSB & DSB \\
\hline $1.4 \mathrm{~nm}+$ AuNP & $(9.3 \pm 0.4) \times 10^{-8}$ & $(2.2 \pm 0.3) \times 10^{-9}$ & $1.4 \pm 0.2$ & $1.2 \pm 0.3$ \\
$30 \mathrm{~nm}+$ AuNP & $(8.3 \pm 0.2) \times 10^{-8}$ & $(2.9 \pm 0.3) \times 10^{-9}$ & $1.3 \pm 0.2$ & $1.5 \pm 0.4$ \\
$30 \mathrm{~nm}-$ AuNP & $(6.1 \pm 0.1) \times 10^{-8}$ & $(1.5 \pm 0.2) \times 10^{-9}$ & $0.9 \pm 0.1$ & $0.8 \pm 0.2$ \\
Control & $(6.7 \pm 0.9) \times 10^{-8}$ & $(1.9 \pm 0.5) \times 10^{-9}$ & 1.0 & 1.0 \\
\hline
\end{tabular}

Notes: * $\mathrm{Da}$ (Dalton) is the unit for molecular weight of DNA, equivalent to atomic mass units. **Dose enhancement factor $=$ SSB or DSB yield with treatment/yield of control.

\section{ROS Generation by AuNPs}

Figure 6 shows the fluorescence intensity of APF as a function of radiation dose in the presence of AuNPs. APF intensity reflects ROS yields, particularly those of hydroxyl radicals $(\bullet \mathrm{OH})(9)$. ROS yields almost linearly increased with the delivered dose by ${ }^{192} \operatorname{Ir} \gamma$-rays. There were no significant differences between ROS yields in the presence of +AuNPs $(30 \mathrm{~nm})$ and -AuNPs $(30 \mathrm{~nm})$. However, ROS yields were considerably smaller in the presence of +AuNPs $(1.4 \mathrm{~nm})$ than AuNPs $(30 \mathrm{~nm})$.

\section{Discussion}

\section{Effect of Radiosensitization by + AuNPs on DNA Damage}

SSB and DSB yields increased in plasmid DNA irradiated by ${ }^{192} \mathrm{Ir} \gamma$-rays in the presence of $30 \mathrm{~nm}+$ AuNPs (Figures 4

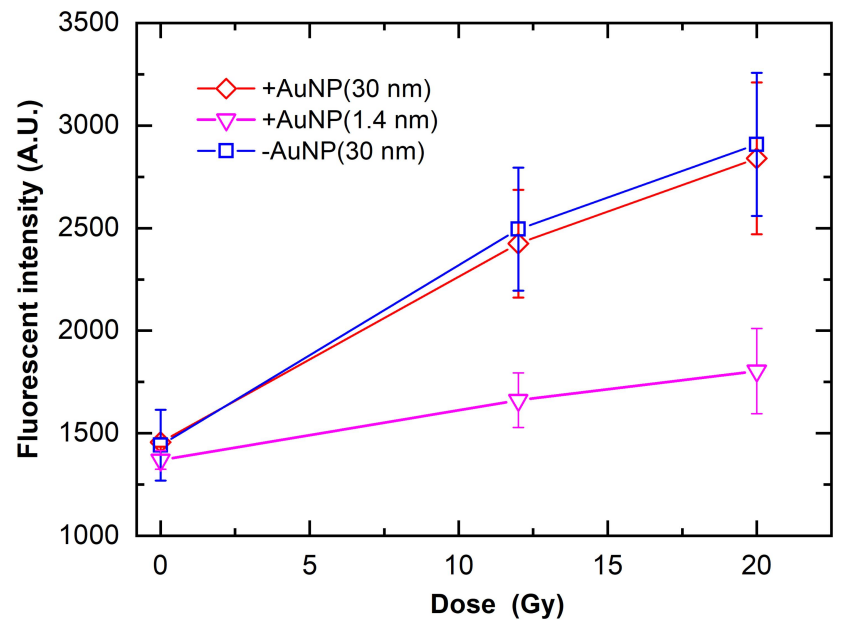

Figure 6 Yields of reactive oxygen species (ROS) as a function of radiation dose irradiated with the ${ }^{192} \mathrm{Ir}$ source in the presence of gold nanoparticles (AuNPs). ROS yields are evaluated as the fluorescence intensity of the fluorescent probe sensitive to ROS yields. 
and 5). Plasmid damage yields appear to be highly dependent on the scavenging capacity of the preparation buffer, such as Tris. ${ }^{21}$ Variations in the fractions of SC and L DNA with dose are similar trends, as observed in previous DNA studies using the same buffer $(0.25 \mathrm{mM}$ TE) with $13 \mu \mathrm{g} / \mathrm{mL}$ AuNP and 25.6 keV X-rays. ${ }^{21}$ The increases observed in SSBs were similar for both $1.4 \mathrm{~nm}$ and $30 \mathrm{~nm}+$ AuNPs. In contrast, DSBs increased more in the presence of $30 \mathrm{~nm}+\mathrm{AuNPs}$ than 1.4 nm + AuNPs (Figure 5).

+ AuNPs exerted a radiosensitization effect on plasmid DNA damage induced by the ${ }^{192} \mathrm{Ir} \gamma$-rays used in HDR brachytherapy. As a result of ${ }^{192} \operatorname{Ir} \gamma$-ray irradiation in HDR brachytherapy, the DEFs were 1.3 for SSBs and 1.5 for DSBs for $30 \mathrm{~nm}+$ AuNPs, respectively (Table 1). The DEFs had relatively larger uncertainty because the DEFs were calculated from damage yields with/without AuNPs and suffered from error propagation of these values. Our data for SSB yield enhancement with medium-energy photons are comparable to values obtained by lowenergy photons using the same buffer (0.25 mM Tris). The reported DEF was $\sim 1.5$ for SSBs using $160 \mathrm{kVp}$ X-rays with $13 \mu \mathrm{g} / \mathrm{mL}(1.25 \mathrm{nM})$ and 11.9-nm diameter AuNPs. $^{21}$ Our values are also comparable to those obtained by Butterworth et al using $160 \mathrm{kVp} \mathrm{X}$-rays in Tris-EDTA buffer, which has more scavenging capacity than the buffer used in this study. ${ }^{20}$ Their results showed that the dose modifying factors were 2.12 (SSB) and 1 (DSB) for $50 \mu \mathrm{g} / \mathrm{mL} 20 \mathrm{~nm}$ AuNPs and 2.29 (SSB) and 1.25 (DSB) for $50 \mu \mathrm{g} / \mathrm{mL} 5 \mathrm{~nm}$ AuNPs. Brun et al reported that DEFs are 1.5-2.0 for SSBs in the presence of 20-40 nm AuNPs irradiated with $49 \mathrm{keV}$ X-rays in water. ${ }^{22}$ Morozow et al reported that DEFs are 1.91-2.74 for SSBs in the presence of $26 \mathrm{~nm}$ AuNPs $(2.4 \mathrm{mg} / \mathrm{mL})$ irradiated with $100-200 \mathrm{kVp} \mathrm{X}$-rays. ${ }^{23}$ All these previous works used non-functionalized AuNPs did not explicitly specify the surface charge of AuNPs used. These AuNPs presumably possess negatively charged due to citrate ligands, which remained after the synthesis. DEF for SSBs by +AuNPs was consistent with previously reported values obtained with -AuNPs. Foley et al used $5 \mathrm{~nm}$ + AuNPs and reported that dose enhancement $\sim 2.1$ at 1 Gy for SSBs by $100 \mathrm{keV}$ X-rays. ${ }^{24}$ The DEFs obtained in this study are smaller than those obtained using lowenergy X-rays. These results are consistent with the knowledge that lower energy photons interact with AuNPs through photoelectric absorption more than medium-energy photons and provide larger dose enhancement.
Burn et al reported that AuNP size dependency on the dose enhancement, as DEF for SSBs increased from 1.2 to 3.0 with the AuNP size increase, within a range of 8-92 $\mathrm{nm}$ in water. ${ }^{22}$ The trends were opposite to those reported by Butterworth et al in TE buffers. ${ }^{20}$ Our data of DEF for SSBs did not show a significant difference between $1.4 \mathrm{~nm}$ and $30 \mathrm{~nm}$ (Table 1). We think that the different trends observed in the three reports could be explained by the difference in experimental conditions, such as different solutions used in each assay.

The DEFs obtained via the plasmid DNA assays are close to the values reported for cell survival assays using the ${ }^{192}$ Ir source (HDR brachytherapy). Shahhoseini et al used $197 \mu \mathrm{g} / \mathrm{mL}$ AuNPs with $15 \mathrm{~nm}$ and reported a DEF of 1.54 for lung cancer cells and 1.67 for prostate cancer cells using the ${ }^{192}$ Ir source. ${ }^{16}$ Retif et al reviewed and summarized the dose modifying factors (reciprocal of DEF) for cell survival and discussed its photon energy dependence. ${ }^{8}$ Our data is also similar to that of the factors for cells using $\mathrm{kV}$ and MV X-rays, $2.4 \mu \mathrm{g} / \mathrm{mL}$ AuNP with $1.9 \mathrm{~nm}$ was used. $^{14}$ The DEFs obtained in this study were similar to those predicted by MC calculations, which demonstrated a DEF of 1.2 using 7-30 mg/mL AuNPs with $1.9 \mathrm{~nm}^{17}$ and $100 \mathrm{~nm} .{ }^{19}$

It is notable that similar dose enhancement by + AuNPs was observed in this study. However, this dose enhancement was achieved using a much lower concentration of AuNPs. For comparison, the concentration of +AuNPs used in this study is approximately 200-3000 times lower than that used in previous DNA assays ${ }^{20,21}$ and cell survival assays, ${ }^{16}$ and six orders of magnitude lower compared with assumptions based on MC calculations. ${ }^{17,19}$

\section{ROS Generation by AuNPs}

ROS generation increased with the delivered dose of AuNPs (Figure 6). APF is known to be particularly sensitive to hydroxyl radicals $(\bullet \mathrm{OH})$, and fluorescence intensity is linearly proportional to $[\bullet \mathrm{OH}] .^{9}$ The ROS generation induced by ${ }^{192} \operatorname{Ir} \gamma$-rays is comparable to previously reported for $100 \mathrm{kVp} \mathrm{X-rays.}{ }^{9}$ An increase in fluorescence intensity reflects increasing ROS in the presence of AuNPs.

The results for the $1.4 \mathrm{~nm}$ and $30 \mathrm{~nm}+$ AuNPs showed that AuNPs that generated higher amounts of ROS had a larger dose enhancement effect on DNA damage (Figure 5). An increase in SSBs and DSBs (ie dose enhancement by +AuNPs) could be explained by an increase in ROS generation by AuNPs irradiated with ${ }^{192} \operatorname{Ir} \gamma$-rays. 
AuNP-induced ROS enhancement increases SSB and DSB yields. In contrast, the ROS yield of $1.4 \mathrm{~nm}+\mathrm{AuNPs}$ is smaller than that of $30 \mathrm{~nm}+$ AuNPs at the same dose (Figure 6). These trends contradict previous findings that smaller AuNPs yield more ROS than larger AuNPs due to their larger surface area. ${ }^{9}$ However, previous findings are based on negatively charged AuNPs, which are much more stable against aggregations. There was a decrease in ROS yield when $1.4 \mathrm{~nm}+$ AuNPs were used, presumably due to AuNP aggregation. This was further confirmed via TEM images of $1.4 \mathrm{~nm}+$ AuNPs.

In contrast, the ROS yield did not differ between similarly sized AuNPs, such as $30 \mathrm{~nm}+$ AuNPs and of $30 \mathrm{~nm}$-AuNPs. The amount of ROS generated by ${ }^{192}$ Ir $\gamma$ ray irradiation was similar for both $30 \mathrm{~nm}+$ AuNPs and 30 $\mathrm{nm}$-AuNPs and was independent of the surface charge of the AuNPs.

\section{Effects of Surface Charge of AuNPs}

The surface charge of the AuNPs did not change the amount of ROS generated (Figure 6). While both + AuNPs and -AuNPs yielded similar levels of ROS, the extent to which they increased DNA damage differed. The DEF for $30 \mathrm{~nm}+$ AuNPs was $1.3 \pm 0.2$ for SSBs and $1.5 \pm$ 0.4 for DSBs (Table 1). In contrast, there was no significant dose enhancement observed in the presence of -AuNPs, and damage yields were similar to those observed in the control group. Only the addition of + AuNPs promoted SSBs and DSBs in the plasmids.

We used similar sizes and concentrations of +AuNPs and -AuNPs, but their surface charges differed. The effect of AuNPs on radiation-induced DNA damage was, therefore, affected by the surface properties of the AuNPs. One possible explanation of these differences in DNA damage sensitization by these surface charges is given as follows: As DNA has a net negative charge, + AuNPs tend to be attracted and attach to the DNA. Binding of positive AuNPs to negative DNA was essential for dose enhancement. Accordingly, the interaction between DNA and + AuNPs, such as the binding and/or local increase of + AuNPs around the DNA, results in differences in DNA radiosensitization to radiation-induced damage compared to the radiosensitization induced by -AuNPs.

ROS were generated by the secondary electrons produced by the ${ }^{192} \operatorname{Ir} \gamma$-rays absorbed by the AuNPs. These electrons have enough energy to travel away from AuNPs and produce ROS. Thus, even though electrons have a negative charge, ROS generation is not affected by the surface charge of AuNPs.

MC calculations predicted that AuNPs would induce a DEF of $\sim 1.2$ in tumors treated with ${ }^{192} \operatorname{Ir} \gamma$-rays. However, these calculations assumed the use of very high concentrations of AuNPs (10-100 mg/g) ${ }^{17-19}$ which is an unrealistic concentration for clinical applications. Thus, a strategy to reduce the concentration of AuNPs while providing significant dose enhancement is necessary for their clinical application. In this study, +AuNPs were successfully used to considerably reduce AuNP concentrations by approximately three to six orders of magnitude, while providing similar dose enhancement. Our study, therefore, presents a promising strategy for increasing the local concentration of AuNPs around DNA by targeting DNA with positively charged AuNPs. We found that a low concentration of + AuNPs effectively promoted radiosensitization for DNA damage induced by ${ }^{192} \operatorname{Ir} \gamma$-rays. This radiosensitization occurred via an increase of the local concentration of +AuNPs around the DNA to a greater extent than-AuNPs.

We assume the use of +AuNPs with brachytherapy for the treatment of cervix cancer. For drug delivery, one of the strategies we suggest is the direct intratumoral injection of +AuNPs (sizes $<10 \mathrm{~nm}$ ) to the tumor volumes ${ }^{35,36}$ We think that this method provides specific accumulation in tumor cells while avoiding problems in blood circulation, such as renal excretion, capture in liver and spleen. ${ }^{37}$ In studies involving rat having glioma, intratumoral injection of $25 \mathrm{mg} / \mathrm{mL}$ AuNPs improved survival significantly, providing the concentrations of $5 \mathrm{mg} / \mathrm{mL}$ AuNPs in tumor. ${ }^{35}$ This amount of +AuNP could be synthesized by the methods adopted in our study. However, several limitations must be resolved to use +AuNPs in clinical applications.

Firstly, the usage of optimal sizes of +AuNPs needs further investigation. DNA binding ability of +AuNPs could be exploited and methods for their efficient delivery to the tumors should be studied. ${ }^{35}$ We think that $1.4 \mathrm{~nm}$ + AuNPs could be attracted by the DNA in vivo; however, $30 \mathrm{~nm}+$ AuNPs could not directly reach the DNA inside the cell nucleus. + AuNPs should be smaller than $\sim 5 \mathrm{~nm}$ of the nuclear pore complex, which is present on the envelope of the cell nucleus and allows the transport of molecules. ${ }^{38}$ Thus, we think that the sizes of +AuNPs smaller than $5 \mathrm{~nm}$ would be preferable for future studies.

The second issue was the drug delivery of +AuNPs to targets. ${ }^{35}$ While +AuNPs target DNA, they should also 
target cancer cells and pass through the cell nucleus before reaching genomic DNA in clinical applications. We think that it is possible for bare +AuNP, without further modification, to reach the target cell. Cho et al reported that + AuNPs were adsorbed on the cell membrane (negatively charged) and showed a higher level (5-10 times) of uptake by the cell compared to -AuNPs. ${ }^{39}$ Interestingly, they suggested that +AuNPs can directly pass through the cell membrane without mediation by the endocytotic pathway.

Another method to deliver +AuNPs to cancer cells is by using liposomes: +AuNPs (size below $5 \mathrm{~nm}$ ) are contained in liposomes, which causes an increase in uptake by the cell through endocytosis. ${ }^{40}$ Then, +AuNPs is released into the cytoplasm, and should then be transported to the nucleus of the cells to bind genomic DNA. This could be achieved by their affinity to DNA because of having a positive charge, or modifying the nuclear transportation signals on the surface of +AuNPs. ${ }^{40-42}$ Furthermore, multistage drug delivery systems are proposed, which change their physicochemical properties (such as size, surface charge) to achieve tumor penetration, enhanced cellular uptake, in response to the differences in the physiological blood circulation, tumor microenvironment, and intracellular environment. ${ }^{43}$

Thirdly, the stability of +AuNPs must be improved, especially for those with smaller sizes below $5 \mathrm{~nm}$. + AuNPs are relatively unstable compared to -AuNPs. + AuNPs are known to be unstable, particularly in high salt conditions, and easily aggregate under physiological conditions. Thus, aggregation must be prevented, potentially via the modification of blocking reagents. Surface or amine group modifications are necessary to overcome or reduce these disadvantages caused by positive charges.

\section{Conclusion}

In this study, we investigated the ability of +AuNPs to enhance plasmid DNA damage induced by ${ }^{192} \operatorname{Ir} \gamma$-rays in HDR brachytherapy. +AuNPs promote a comparable level of radiosensitization to both SSBs and DSBs at lower concentrations than those used in cell survival assays and MC calculations. The ability of +AuNPs to promote plasmid DNA damage is due to enhanced ROS generation, such as $\bullet \mathrm{OH}$ radicals. While, - AuNPs generated similar levels of ROS, they did not show significant DNA damage enhancement ability. Thus, dose enhancement by low concentrations of +AuNPs presumably occurred via binding DNA or increasing the local concentration around DNA. +AuNPs are effective candidate radiosensitizers that can be used in conjunction with HDR brachytherapy. Future development of surface modifications for improved stability and tumor delivery would generate methods that can more widely apply + AuNPs alongside therapeutic ${ }^{192} \mathrm{Ir} \gamma$-rays in HDR brachytherapy.

\section{Abbreviations}

AuNPs, gold nanoparticles; DEF, dose enhancement factor; DSB, double-stranded breaks; HDR, high-dose rate; MC, Monte Carlo; ROS, reactive oxygen species; SSB, singlestranded breaks; TEM, transmission electron microscopy.

\section{Acknowledgments}

This work was supported in part by JSPS KAKENHI Grant Numbers 25870707 and JP18K07679, the Program of the Network-Type Joint Usage/Research Center for Radiation Disaster Medical Science of Hiroshima University, and the NU-AIST alliance project. We are grateful to Saori Yamamoto, Mirano Kakinuma, and Takayuki Tsuchinoto from Kitasato University and Yuzuki Nishihara from Nagoya University for providing experimental support.

\section{Disclosure}

The authors declare no conflict of interests in this work.

\section{References}

1. Kubo HD, Glasgow GP, Pethel TD, et al. High dose-rate brachytherapy treatment delivery: report of the AAPM radiation therapy committee task group no. 59. Med Phys. 1998;25(4):375-403. doi:10.1118/ 1.598232

2. Nag S, Cardenes H, Chang S, et al. Proposed guidelines for image-based intracavitary brachytherapy for cervical carcinoma: report from image-guided brachytherapy working group. Int J Radiat Oncol Biol Phys. 2004;60(4):1160-1172. doi:10.1016/j.ijrobp.2004.04.032

3. Pötter R, Haie-Meder C, Van Limbergen E, et al. Recommendations from gynaecological (GYN) GEC ESTRO working group (II): concepts and terms in 3D image-based treatment planning in cervix cancer brachytherapy $-3 \mathrm{D}$ dose volume parameters and aspects of 3D imagebased anatomy, radiation physics, radiobiology. Radiother Oncol. 2006;78(1):67-77. doi:10.1016/j.radonc.2005.11.014

4. Yoshida K, Yamazaki H, Takenaka T, et al. A dose-volume analysis of magnetic resonance imaging-aided high-dose-rate image-based interstitial brachytherapy for uterine cervical cancer. Int J Radiat Oncol Biol Phys. 2010;77(3):765-772. doi:10.1016/j.ijrobp.2009.05.027

5. Lessard E, Pouliot J. Inverse planning anatomy-based dose optimization for HDR-brachytherapy of the prostate using fast simulated annealing algorithm and dedicated objective function. Med Phys. 2001;28(5):773-779. doi:10.1118/1.1368127

6. Yoshio K, Murakami N, Morota M, et al. Inverse planning for combination of intracavitary and interstitial brachytherapy for locally advanced cervical cancer. J Radiat Res. 2013;54(6):1146-1152. doi: $10.1093 / \mathrm{jrr} / \mathrm{rrt} 072$ 
7. Hainfeld JF, Dilmanian FA, Slatkin DN, Smilowitz HM. Radiotherapy enhancement with gold nanoparticles. $J$ Pharm Pharmacol. 2008;60(8):977-985. doi:10.1211/jpp.60.8.0005

8. Retif P, Pinel S, Toussaint M, et al. Nanoparticles for radiation therapy enhancement: the key parameters. Theranostics. 2015;5 (9):1030-1044. doi:10.7150/thno.11642

9. Misawa M, Takahashi J. Generation of reactive oxygen species induced by gold nanoparticles under $\mathrm{x}$-ray and UV irradiations. Nanomedicine. 2011;7(5):604-614. doi:10.1016/j.nano.2011.01.014

10. Hainfeld JF, Slatkin DN, Smilowitz HM. The use of gold nanoparticles to enhance radiotherapy in mice. Phys Med Biol. 2004;49(18): N309-15. doi:10.1088/0031-9155/49/18/N03

11. Butterworth KT, Coulter JA, Jain S, et al. Evaluation of cytotoxicity and radiation enhancement using $1.9 \mathrm{~nm}$ gold particles: potential application for cancer therapy. Nanotechnology. 2010;23 (29):295101. doi:10.1088/0957-4484/21/29/295101

12. Hainfeld JF, Smilowitz HM, O'Connor MJ, Dilmanian FA, Slatkin DN. Gold nanoparticle imaging and radiotherapy of brain tumors in mice. Nanomedicine. 2013;8(10):1601-1609. doi:10.2217/ nnm. 12.165

13. Chithrani DB, Jelveh S, Jalali F, et al. Gold nanoparticles as radiation sensitizers in cancer therapy. Radiat Res. 2010;173(6):719-728. doi:10.1667/RR1984.1

14. Jain S, Coulter JA, Hounsell AR, et al. Cell-specific radiosensitization by gold nanoparticles at megavoltage radiation energies. Int J Radiat Oncol Biol Phys. 2011;79(2):531-539. doi:10.1016/j. ijrobp.2010.08.044

15. Laprise-Pelletier M, Simão T, Fortin MA. Gold nanoparticles in radiotherapy and recent progress in nanobrachytherapy. Adv Healthc Mater. 2018;7(16):e1701460. doi:10.1002/adhm.201701460

16. Shahhoseini E, Ramachandran P, Patterson WR, Geso M. Determination of dose enhancement caused by AuNPs with Xoft ${ }^{\mathbb{B}}$ Axxent ${ }^{\circledR}$ Electronic $\left(\mathrm{eBx}^{\mathrm{TM}}\right)$ and conventional brachytherapy: in vitro study. Int J Nanomed. 2018;13:5733-5741. doi:10.2147/IJN.S174624

17. Cho SH, Jones BL, Krishnan S. The dosimetric feasibility of gold nanoparticle-aided radiation therapy (GNRT) via brachytherapy using low-energy gamma-/x-ray sources. Phys Med Biol. 2009;54 (16):4889-4905. doi:10.1088/0031-9155/54/16/004

18. Lechtman E, Chattopadhyay N, Cai Z, Mashouf S, Reilly R, Pignol JP. Implications on clinical scenario of gold nanoparticle radiosensitization in regards to photon energy, nanoparticle size, concentration and location. Phys Med Biol. 2011;56(15):4631-4647. doi:10.1088/0031-9155/56/15/001

19. Zabihzadeh M, Arefian S. Tumor dose enhancement by nanoparticles during high dose rate 192Ir brachytherapy. J Cancer Res Ther. 2015;4 (4):752-759. doi:10.4103/0973-1482.153668

20. Butterworth KT, Wyer JA, Brennan-Fournet M, et al. Variation of strand break yield for plasmid DNA irradiated with High-Z metal nanoparticles. Radiat Res. 2008;170(3):381-387. doi:10.1667/ RR1320.1

21. McMahon SJ, Hyland WB, Brun E, et al. Dependence of gold nanoparticle radiosensitization in plasmid DNA. Phys Chem. 2011;115:20160-20167.

22. Brun E, Sanche L, Sicard-Roselli C. Parameters governing gold nanoparticle X-ray radiosensitization of DNA in solution. Colloids Surf B Biointerfaces. 2009;72(1):128-134. doi:10.1016/j.colsurfb.2009.03.025

23. Morozov KV, Kolyvanova MA, Kartseva ME, et al. Radiosensitization by gold nanoparticles: impact of the size, dose rate, and photon energy. Nanomaterials. 2020;10(5):952. doi:10.3390/nano10050952

24. Foley EA, Carter JD, Shan F, et al. Enhanced relaxation of nanoparticle-bound supercoiled DNA in X-ray radiation. Chem Commun. 2005;25(25):3192-3194. doi:10.1039/b503425f

25. Niidome T, Nakashima K, Takahashi H, Niidome Y. Preparation of primary amine-modified gold nanoparticles and their transfection ability into cultivated cells. Chem Commun (Camb). 2004;17 (17):1978-1979. doi:10.1039/b406189f
26. Zheng S, Newton GL, Ward JF, Fahey RC. Aerobic radioprotection of pBR322 by thiols: effect of thiol net charge upon scavenging of hydroxyl radicals and repair of DNA radicals. Radiat Res. 1992;130 (2):183-193. doi:10.2307/3578275

27. Yogo K, Murayama C, Fujisawa Y, et al. Potential mechanisms for protective effect of D-methionine on plasmid DNA damage induced by therapeutic carbon ions. Radiat Res. 2020;193(6):513-519. doi:10.1667/RR15502.1

28. Nath R, Anderson LL, Luxton G, Weaver KA, Williamson JF, Meigooni AS. Dosimetry of interstitial brachytherapy sources: recommendations of the AAPM radiation therapy committee task group no. 43. Med Phys. 1995;22(2):209-234. doi:10.1118/1.597458

29. Rivard MJ, Coursey BM, DeWerd LA, et al. Update of AAPM task group no. 43 report: a revised AAPM protocol for brachytherapy dose calculations. Med Phys. 2004;31(3):633-674. doi:10.1118/1.1646040

30. Hanai R, Yazu M, Hieda K. On the experimental distinction between SSBs and DSBs in circular DNA. Int $J$ Radiat Biol. 1998;73 (5):475-479. doi:10.1080/095530098142013

31. Yogo K, Ogawa T, Hayashi M, Harada Y, Nishizaka T, Kinosita JK. Direct observation of strand passage by DNA-topoisomerase and its limited processivity. PLoS One. 2012;7(4):e34920. doi:10.1371/journal.pone. 0034920

32. Usami N, Kobayashi K, Furusawa Y, Frohlich H, Lacombe S, Le Sech C. Irradiation of DNA loaded with platinum containing molecules by fast atomic ions C6+ and Fe 26+. Int J Radiat Biol. 2007;83 (9):569-576. doi:10.1080/09553000701447130

33. Usami N, Kobayashi K, Hirayama R, Furusawa Y, Porcel E, Lacombe S. Comparison of DNA breaks at entrance channel and bragg peak induced by fast $\mathrm{C} 6+$ ions-influence of the addition of platinum atoms on DNA. J Radiat Res. 2010;51(1):21-26. doi:10.1269/jrr.09035

34. Lloyd RS, Haidle CW, Robberson DL. Bleomycin-specific fragmentation of double-stranded DNA. Biochemistry. 1978;17 (10):1890-1896. doi:10.1021/bi00603a014

35. Bobyk L, Edouard M, Deman P, et al. Photoactivation of gold nanoparticles for glioma treatment. Nanomedicine. 2013;9 (7):1089-1097. doi:10.1016/j.nano.2013.04.007

36. Shi M, Paquette B, Thippayamontri T, et al. Increased radiosensitivity of colorectal tumors with intra-tumoral injection of low dose of gold nanoparticle. Int $J$ Nanomed. 2016;11:5323-5333. doi:10.2147/IJN. S97541

37. Nam J, Won N, Bang J, et al. Surface engineering of inorganic nanoparticles for imaging and therapy. Adv Drug Deliv Rev. 2013;65(5):622-648. doi:10.1016/j.addr.2012.08.015

38. Lechtman E, Pignol J-P. Interplay between the gold nanoparticle sub-cellular localization, size, and the photon energy for radiosensitization. Sci Rep. 2017;7(1):13268. doi:10.1038/s41598017-13736-y

39. Cho EC, Xie J, Wurm PA, et al. Understanding the role of surface charges in cellular adsorption versus internalization by selectively removing gold nanoparticles on the cell surface with a $\mathrm{I} / \mathrm{KI}$ etchant. Nano Lett. 2009;9(3):1080-1084. doi:10.1021/nl803487r

40. Nativo P, Prior IA, Brust M. Uptake and intracellular fate of surface-modified gold nanoparticles. ACS Nano. 2008;2 (8):1639-1644. doi:10.1021/nn800330a

41. Ryan JA, Overton KW, Speight ME, et al. Cellular uptake of gold nanoparticles passivated with BSA-SV40 large T antigen conjugates. Anal Chem. 2007;79(23):9150-9159. doi:10.1021/ac0715524

42. Özçelik S, Pratx G. Nuclear-targeted gold nanoparticles enhance cancer cell radiosensitization. Nanotechnology. 2020;31(41):415102. doi:10.1088/1361-6528/aba02b

43. Chen B, Dai W, He B, et al. Current multistage drug delivery systems based on the tumor microenvironment. Theranostics. 2017;7 (3):538-558. doi:10.7150/thno.16684 


\section{Publish your work in this journal}

The International Journal of Nanomedicine is an international, peerreviewed journal focusing on the application of nanotechnology in diagnostics, therapeutics, and drug delivery systems throughout the biomedical field. This journal is indexed on PubMed Central, MedLine, CAS, SciSearch ${ }^{\mathbb{R}}$, Current Contents ${ }^{(\mathbb{R}} /$ Clinical Medicine, $^{-}$
Journal Citation Reports/Science Edition, EMBase, Scopus and the Elsevier Bibliographic databases. The manuscript management system is completely online and includes a very quick and fair peer-review system, which is all easy to use. Visit http://www.dovepress.com/ testimonials.php to read real quotes from published authors.

Submit your manuscript here: https://www.dovepress.com/international-journal-of-nanomedicine-journal 